\title{
Frenetic Bounds on the Entropy Production
}

\begin{abstract}
Christian Maes
Instituut voor Theoretische Fysica, KU Leuven, Celestijnenlaan 200D, B-3001 Leuven, Belgium

(Received 21 May 2017; revised manuscript received 29 August 2017; published 17 October 2017)

We give a systematic derivation of positive lower bounds for the expected entropy production (EP) rate in classical statistical mechanical systems obeying a dynamical large deviation principle. The logic is the same for the return to thermodynamic equilibrium as it is for steady nonequilibria working under the condition of local detailed balance. We recover there recently studied "uncertainty" relations for the EP, appearing in studies about the effectiveness of mesoscopic machines. In general our refinement of the positivity of the expected EP rate is obtained in terms of a positive and even function of the expected current(s) which measures the dynamical activity in the system, a time-symmetric estimate of the changes in the system's configuration. Also underdamped diffusions can be included in the analysis.
\end{abstract}

DOI: 10.1103/PhysRevLett.119.160601

The entropy production (EP) rate $\sigma(\rho, j)$ appears in the entropy balance equation within irreversible thermodynamics as a bilinear form of forces and currents [1]. Forces depend on the density $\rho$ and the current $j$ indicates the displacements in the system. Without external driving and under local equilibrium, we have, e.g.,

$$
\begin{aligned}
\sigma(\rho, j) & =\int_{\Omega} d X \nabla \frac{\delta S}{\delta \rho(X)} j(X)=\int_{\Omega} d X \frac{\delta S}{\delta \rho(X)} \dot{\rho}(X) \\
& =\frac{d}{d t} S[\rho(X), X \in \Omega],
\end{aligned}
$$

where we took a locally conserved field $\rho(X)$ with $j(X)$ the local current in a region $\Omega$ with periodic boundary conditions, and $\nabla j=-\dot{\rho}$. Then, for all possible $(\rho, j)$ the EP rate is the time derivative of the entropy $S$ and the force is the gradient of the variational derivative $[\delta S / \delta \rho(X)]$. In the case of steady driven systems, the first line in Eq. (1) gets replaced by

$$
\sigma(\rho, j)=\int d X F(\rho(X), X) j(X),
$$

where the force $F$ is now not globally derivable from a thermodynamic potential. The state $\rho$ is often representing the profile of energy or particles in a macroscopic system but it could also be the single particle phase space density on $X=(q, p)$ for underdamped colloidal motion or the density of independent random walkers at the sites of a given graph, in which case we sum over its edges $\{x, y\}$,

$\sigma(\rho, j)=\sum j(x, y) F(x, y), F(x, y)=\log \frac{\rho(x) k(x, y)}{\rho(y) k(y, x)}$

for transition rates $k(x, y)$; see, e.g., Ref. [2]. Here we focus on open systems because we want to include stationary driven systems, and the EP is the change of the total entropy in system plus environment.
The expected EP rate $\sigma^{*}(\rho)$ given the present state $\rho$ of the system is always non-negative, which is a version of the second law of thermodynamics. We are interested here in informative lower bounds for $\sigma^{*}$, i.e., for $\sigma^{*}=\sigma\left(\rho, j^{*}(\rho)\right)$ with $j^{*}$ the expected current at $\rho$.

The lower bounds we derive in the present Letter are obtained from the dynamical activity in the system. That notion is rather new and does not appear in standard irreversible thermodynamics except in terms of linear response coefficients. Yet the first intuition is simple enough: to have a transition between entropically different macroscopic conditions there needs to be some timesymmetric exploration of state space too. That dynamical activity consists of two related parts: (i) the escape rate from the condition $\rho$, and (ii) the frenetic motion or undirected currents of the microscopic degrees of freedom within condition $\rho$. Interestingly, that provides a conceptual and mathematical complement to entropic changes as the dynamical activity indicates time-symmetric changes, both in the exit or access of states as in being inversely proportional to the residence or sojourn time in a macroscopic condition. In Boltzmann's picture of relaxation to equilibrium in terms of a flow on phase space visiting the various regions of macrostates, it adds "surface" to "volume" considerations, as it refers to the accessibility of and the escapeability from macroscopic conditions and not only to their entropy; see the cartoon in Fig. 1.

The starting point of our analysis is dynamical fluctuation theory [3-5]. That means we consider the probability of empirically possible trajectories, specified at times $s \in[0, t]$ via a variable density $\rho_{s}$ and a current $j_{s}$. Those $\rho_{s}$ can be fields on physical space or they can be empirical averages of particle properties, etc. The empirical rate of change in time is given by the current $j_{s}$, which often obeys a further constraint such as in the continuity equation $\dot{\rho}_{s}+\mathcal{D} j_{\rho_{s}}=0$, where $\mathcal{D}$ is a divergence, but other operators $\mathcal{D}$ are possible in case $\rho_{s}$ does not refer to a locally conserved quantity. We repeat that $\left(\rho_{s}, j_{s}\right), s \in[0, t]$, 


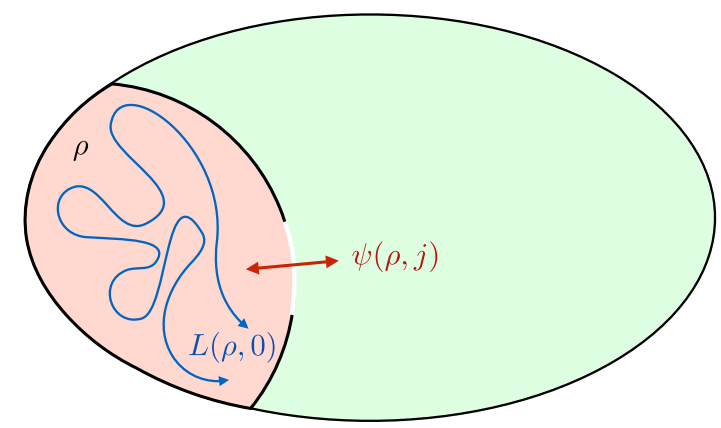

FIG. 1. Entropy production is bounded from below by two types of activity. For moving into a larger phase volume from condition $\rho$, thereby producing entropy, the dynamics must be sufficiently active inside $\rho$, represented by $L(\rho, 0)$, and must readily find an escape route, represented by $\psi(\rho, j)$.

denotes a possible density-current trajectory on some level of coarse graining, realizable from the microscopic laws, constituents, and initial conditions. The probability of such a trajectory then satisfies

$$
\operatorname{Prob}\left[\left(\rho_{s}, j_{s}\right), 0 \leq s \leq t\right] \simeq e^{-N \mathcal{F}\left(\rho_{0}\right)} e^{-N \int_{0}^{t} d s L\left(\rho_{s}, j_{s}\right)} .
$$

Here, $N \uparrow+\infty$ is a scale parameter like the number of independent copies of a finite Markov process, or the number of particles, the volume, etc., where Eq. (4) must be understood as giving the asymptotic exponential behavior of probabilities. In fact, Eq. (4) is the formal extension of the Boltzmann-Planck-Einstein macroscopic fluctuation theory to the time domain $[3,4]$, where now the trajectory $\left(\rho_{s}, j_{s}\right), s \in[0, t]$ is the random variable. The functional $\mathcal{F}$ in Eq. (4) is a thermodynamic potential (typically in units of $\left.k_{B} T\right)$ when the Prob refers to the thermodynamic equilibrium distribution at temperature $T$. For closed isolated systems in equilibrium, $\mathcal{F}=-S$ is minus the entropy of Eq. (1). If in stationary nonequilibrium the $\mathcal{F}$ is still sometimes called a nonequilibrium free energy to emphasize the analogy. The Lagrangian $L$ in Eq. (4) further determines the plausibility of the various possible trajectories. Note that the Lagrangian $L$ in Eq. (4) is essentially constructed and explicitly known when starting from a (semi-)Markov process [6-10]. That is part of the mathematical work that started with the large deviation theory for Markov processes in Refs. [5,11,12].

Properties that we assume from the outset are that $L \geq 0$ and is convex in $j$ for all $\rho$. Note that due to the large $N$ the weights in Eq. (4) are exponentially small with respect to the zero-cost flow $j_{s}^{*}=j^{*}\left(\rho_{s}\right)$, which solves $L\left(\rho_{s}, j_{s}^{*}\right)=0$ for all times $s$ and induces a unique evolution equation $\dot{\rho}_{s}+\mathcal{D} j_{s}^{*}=0$. Under the present assumptions, finding the typical trajectory is thus equivalent with finding for any given $\rho$ the solution $j^{*}=j^{*}(\rho)$ of $L\left(\rho, j^{*}\right)=0$. To that $j^{*}$ corresponds the expected EP rate $\sigma^{*}=\sigma\left(\rho, j^{*}\right)$, for which we want to obtain bounds as the main goal of this Letter. Note that the unit of time is arbitrary here, and currents (with dimension of frequency) must always be compared with other time constants.

Entropy production and dynamical activity.-An important question in the construction of nonequilibrium statistical mechanics is to identify the phenomenological and operational meaning of the Lagrangian $L(\rho, j)$ in Eq. (4). For that purpose $L$ is most usefully split up in a timesymmetric and a time-antisymmetric part,

$$
L(\rho, j)=\frac{1}{2}[L(\rho, j)+L(\rho,-j)]-\frac{1}{2} \sigma(\rho, j),
$$

where $\sigma(\rho, j)=L(\rho,-j)-L(\rho, j)$ is antisymmetric under time reversal. As the notation already suggests, when the density $\rho$ is determined by time-symmetric variables (being even under kinematic time-reversal), $\sigma(\rho, j)$ is indeed the EP rate per $k_{B}$ corresponding to the couple $(\rho, j)$. That identification in Eq. (5) follows from the condition of the local detailed balance as holds for externally driven systems, or for systems that are in weak contact with multiple equilibrium reservoirs that are sufficiently separated, cf. Refs. $[13,14]$. It is the essential reason behind the well-known symmetries in the distribution of the EP; see also Ref. [15] for more references.

Next comes the time-symmetric part of the Lagrangian, which yields a mathematical identification of the dynamical activity:

$$
L(\rho, j)+L(\rho,-j)=2 \psi(\rho, j)+2 L(\rho, 0) .
$$

There are two contributions as hinted at before, both nonnegative as will turn out. The $L(\rho, 0) \geq 0$ is an (internal) dynamical activity when there is no change in the macroscopic condition $(j=0)$. It corresponds to the activity of the more microscopic degrees of freedom expected at $j=0$ because, as is easy to interpret from Eq. (4) for $\left(\rho_{s}, j_{s}\right)=(\rho, 0), L(\rho, 0)$ is the escape rate from condition $\rho$;

$$
\operatorname{Prob}\left[\left(\rho_{s} \equiv \rho, j_{s} \equiv 0\right), 0 \leq s \leq t\right] \simeq e^{-N \mathcal{F}(\rho)} e^{-N t L(\rho, 0)} .
$$

On the other hand, the $\psi(\rho, j)$ in Eq. (6) gives the dynamical activity corresponding to a given current $j \neq 0$; see Fig. 1 . We have of course $\psi(\rho, j)=\psi(\rho,-j)$, symmetric in $j$ with $\psi(\rho, 0)=0$, and, thus, $\psi$ corresponds to the unoriented traffic between "neighboring" conditions. Moreover, as by construction,

$$
\psi(\rho, j)=L(\rho, j)-L(\rho, 0)+\frac{1}{2} \sigma(\rho, j),
$$

and $\sigma(\rho, j)$ is linear in $j$, it follows that $\psi(\rho, j)$ is convex in $j$, which implies that $\psi(\rho, j) \geq 0$. Together, $\psi(\rho, j)$ and $L(\rho, 0)$ depicted in Fig. 1 inform us about the time-symmetric activity in the system at $(\rho, j)$.

As a final remark about mathematical structure $\psi(\rho, j)$ and $L(\rho, 0)$ are related by Legendre transform. It is indeed easy to check that writing $\sigma(\rho, j)=F j=L(\rho,-j)-L(\rho, j)$, 


$$
2 L(\rho, 0)=\sup _{j}[F j-2 \psi(\rho, j)],
$$

from substituting Eq. (7). Therefore, $0 \leq L(\rho, 0)=$ $\hat{\psi}(\rho, F)$ is the Legendre transform of $\psi(\rho, \cdot)$ at force $F$ and the decomposition (6) is in a pair of convex duals, with current and force being conjugate. For a dynamics with force $F$, the Lagrangian (5) has, therefore, the canonical structure

$$
L(\rho, j)=\psi(\rho, j)+\hat{\psi}(\rho, F)-\frac{1}{2} \sigma(\rho, j) .
$$

Such a structure of joint density-current fluctuations away from equilibrium was developed in Refs. $[6,7,9,16,17]$ to which we refer for more discussion.

Zero-cost flow.-The expected current $j^{*}$ minimizes the action, $L\left(\rho, j^{*}\right)=0$ (zero cost). When $j^{*} \neq 0$, then both $\psi\left(\rho, j^{*}\right)>0$ and $L(\rho, 0)>0$ (because then also force $F \neq 0$ ). On the other hand, combined with Eq. (8) it follows that the expected EP rate $\sigma^{*}=\sigma\left[\rho, j^{*}(\rho)\right]$, function of condition $\rho$ and force $F$ is

$$
\begin{aligned}
\frac{1}{2} \sigma^{*} & =\psi\left(\rho, j^{*}\right)+\hat{\psi}(\rho, F) \\
& =\psi\left(\rho, j^{*}\right)+L(\rho, 0) .
\end{aligned}
$$

That is the crucial identity of this work from which all further results follow. In other words, when we are given macroscopic condition $\rho$, then the expected and, in fact, most likely fate of the system is to take the current $j^{*}(\rho)$ for which the EP rate equals the dynamical activity as in Eq. (9). Macroscopic trajectories can be characterized as those for which at each moment there is a perfect balance between the EP rate and the dynamical activity given the present state. As an immediate consequence follows that every positive lower bound on the dynamical activity [right-hand side of Eq. (9)] implies an interesting lower bound on the expected EP rate, which is the main result, a frenetic bound on the EP rate. Since the right-hand side is strictly positive whenever $j^{*} \neq 0$, Eq. (9) realizes quantitatively the statement that there cannot be a current without EP [18]. Alternatively, we need to have a contribution to motion which is symmetric under time reversal to have strict dissipation. In other words, fluctuations that go either direction, up and down in (variable or path dependent) entropy production must be present for the expected EP rate to be strictly positive.

We can also derive a number of further inequalities which naturally follow from positive lower bounds $\psi(\rho, j) \geq 0$ and $\hat{\psi}(\rho, F) \geq 0$. Both are convex functions for which the Taylor theorem with remainder gives a first simplest bound. Their Hessian matrix is strictly positive in all finite regions around current $j=0$ [for $\psi(\rho, j)]$ or around forcing $F=0$ [for $\hat{\psi}(\rho, F)]$. It is physically natural to assume that there is saturation in both currents and in forces in the sense that there is a maximal possible current amplitude and that all possible forces have a maximal strength. We define then the time constant $m(\rho)$ and frequency $\kappa(\rho)$ from the Hessian matrix of $\psi$ with respect to currents $j$, respectively, of $\hat{\psi}$ with respect to forces $F$ : in the sense of quadratic forms,

$$
m(\rho)=\inf _{j} \operatorname{Hess}[\psi(\rho, j)], \kappa(\rho)=\inf _{F} \operatorname{Hess}[\hat{\psi}(\rho, F)],
$$

where the first infimum is over all currents $j$ with amplitude below $|j| \leq j_{\max }$, a saturation or maximal possible current amplitude $j_{\max }$. Similarly, the second infimum is over all possible forces $F$, which in amplitude are below a maximum $G_{\max }$. We thus get the corollary

$$
\psi\left(\rho, j^{*}\right) \geq \frac{1}{2} m(\rho)\left(j^{*}\right)^{2}, \quad L(\rho, 0)=\hat{\psi}(\rho, F) \geq \frac{1}{2} \kappa(\rho) F^{2},
$$

and, hence,

$$
\sigma^{*} \geq m(\rho)\left(j^{*}\right)^{2}+\kappa(\rho) F^{2}
$$

for $m(\rho), \kappa(\rho)>0$ which measure the quadratic growth of the dynamical activity near $j=0$, respectively, $F=0$. Note that Eq. (10) is the simplest general bound, naturally following from convexity and allowing explicit calculations as we will show. Yet, compared to the universal (9), the bounds (10) are in the spirit of close to equilibrium, where the EP rate indeed becomes quadratic in currents or in forces. For every application there may well be better or more natural bounds and each time (9) carries the promise that those yield bounds on the EP rate. On the other hand, any bound on the expected EP rate $\sigma^{*}$ implies a bound on the dynamical activity.

Examples.-There are various classes of examples and we will not treat the more standard ones. For close-toequilibrium processes or for driven diffusive systems holds the quadratic form

$$
\psi(\rho, j)=\frac{1}{4} \int d X j(X) \Gamma^{-1}[\rho(X)] j(X)
$$

for a symmetric Onsager-matrix $\Gamma$, which depends on the local field. From Eq. (11) it is clear that bounds on the Onsager matrix provide bounds on the dynamical activity, hence, from Eq. (9) bounds on the free energy rate of decay as in Eq. (1), but with $S=-\mathcal{F}$.

The same remains true for overdamped Markov diffusion processes, driven or not. Consider a $d$-dimensional inhomogeneous diffusion, with Itô convention,

$$
\dot{x}_{t}=\chi\left(x_{t}\right) f\left(x_{t}\right)+\nabla \cdot D\left(x_{t}\right)+\sqrt{2 D\left(x_{t}\right)} \xi_{t} .
$$

The mobility $\chi(x)=\beta D(x)$ is a positive $d \times d$ matrix, $\beta>0$ is the inverse bath temperature, and $\xi_{t}$ is standard white noise. Under smoothness and confining boundary 
conditions the Lagrangian is obtained in Ref. [7], and for $\psi(\rho, j)$ we get exactly Eq. (11) with $\Gamma=\rho D$ as indeed the quadratic form is already exact here. Furthermore, there $L(\rho, 0)=\psi\left(\rho, j^{*}\right)$ so that from Eq. (9) the expected EP rate trivially obtains the lower bound,

$$
\sigma^{*} \geq \frac{1}{\max _{x}\|D(x)\|} \int\left[j^{*}(x)\right]^{2} d x .
$$

Things become more interesting for jump processes where nonlinearities are more prominent. Yet all we need remains explicit and we can use the formulas 4.1-4.7 in Ref. [6] to find that for a general Markov jump process with transition rates $\lambda(x, y)$ between states $x \rightarrow y$,

$$
\begin{aligned}
L(\rho, 0)= & \frac{1}{2} \sum_{x \neq y} \frac{\left(j^{*}(x, y)\right)^{2}}{[\sqrt{\rho(x) \lambda(x, y)}+\sqrt{\rho(y) \lambda(y, x)}]^{2}}, \\
\psi(\rho, j)= & \frac{1}{4} \sum_{x \neq y}\left[j(x, y) \log \frac{j(x, y)+\sqrt{j^{2}(x, y)+\gamma(x, y)}}{-j(x, y)+\sqrt{j^{2}(x, y)+\gamma(x, y)}}\right. \\
& \left.+2 \sqrt{\gamma(x, y)}-2 \sqrt{j^{2}(x, y)+\gamma(x, y)}\right]
\end{aligned}
$$

for $\gamma(x, y)=4 \rho(x) \rho(y) \lambda(x, y) \lambda(y, x)=\gamma(y, x)$ measuring time-symmetric reactivity. It is easy enough to check the general inequality $\left(a^{2}-1\right) \log a \geq 2(a-1)^{2}$, when $a>0$, from which it follows by a simple calculation that

$$
\begin{aligned}
\psi(\rho, j) & \geq \frac{1}{4\left(1+\sqrt{1+\alpha^{2}}\right)} \sum_{x \neq y} \frac{j^{2}(x, y)}{\sqrt{j^{2}(x, y)+\gamma(x, y)}} \\
& \geq \frac{1}{4\left(1+\alpha^{2}+\sqrt{1+\alpha^{2}}\right)} \sum_{x \neq y} \frac{j^{2}(x, y)}{\sqrt{\gamma(x, y)}},
\end{aligned}
$$

where $\alpha^{2}:=\max \left[j^{2}(x, y) / \gamma(x, y)\right]$ gives the maximally available normalized current. Thence, Eq. (9) gives a lower bound for the expected EP rate by adding Eqs. (13) and (14).

As an example, take a quantum dot with states $x=0,1$, which we connect to a left and a right load in the Coulomb blockade regime. In chemical language, there are two channels for each transition $x \rightarrow 1-x$. Put $F \geq 0$ (constant) for the potential gradient so that local detailed balance at inverse temperature $\beta$ demands transition rates corresponding to each channel of the following general form,

$$
\begin{array}{ll}
k_{L}(0,1)=\nu e^{\beta F / 2}, & k_{L}(1,0)=\nu e^{-\beta F / 2}, \\
k_{R}(0,1)=\nu e^{-\beta F / 2}, & k_{R}(1,0)=\nu e^{\beta F / 2},
\end{array}
$$

where $\nu=\nu(\beta, F)$ is a reference frequency. We write the densities as $\rho(0)=p=1-\rho(1)$, and the current $j=$ $j(0,1)$ goes from left to right. The Lagrangian was obtained in Ref. [9]. A computation from Eq. (6) yields

$$
\begin{aligned}
L(p, 0)= & 2 \nu\left[\cosh \frac{\beta F}{2}-\sqrt{4 p(1-p)}\right], \\
\psi(p, j)= & j \log \frac{j+\sqrt{4 p(1-p) \nu^{2}+j^{2}}}{-j+\sqrt{4 p(1-p) \nu^{2}+j^{2}}} \\
& +2\left[\sqrt{4 p(1-p) \nu^{2}}-\sqrt{4 p(1-p) \nu^{2}+j^{2}}\right] .
\end{aligned}
$$

We have $L(p, 0) \geq \frac{1}{2} \nu(\beta F)^{2}$, and $\left(\partial^{2} / \partial j^{2}\right) \psi(p, j)=$ $(2 / \nu)\left(1 / \sqrt{4 p(1-p)+(j / \nu)^{2}}\right)$ leads to the time constant $m(p)=2\left[4 p(1-p)+\alpha^{2}\right]^{-1 / 2} / \nu$ in the range $|j / \nu| \leq \alpha$. Therefore, for Eq. (10),

$$
\sigma^{*} \geq \frac{2}{\nu} \frac{1}{\sqrt{4 p(1-p)+\nu^{2} j_{\max }^{2}}}\left(j^{*}\right)^{2}+\beta^{2} \nu F^{2} .
$$

As such, the resulting bounds above add little sensation, but they illustrate the logic and the concepts involved, with the main point that the logic is unchanged from case to case. Various applications to (bio-)physically relevant questions exist; e.g., strictly positive lower bounds for the expected EP rate are interesting for bounds on efficiencies, cf. [19-27]. Yet the systematic bounds of the present Letter show that these bounds are essentially related to time-symmetric activity and fluctuations. It allows further explorations. A case which is absent in the literature is, e.g., the one of underdamped Markov diffusion processes. For processes with degrees of freedom which are odd under kinematic time reversal there exists obviously a condition of generalized detailed balance but the arguments above cannot be applied as such. Suppose indeed quite more general than Eq. (5) that there is a function $h_{\rho}$ so that the Lagrangian satisfies [28]

$$
L\left(\rho, h_{\rho}-j\right)-L\left(\rho, h_{\rho}+j\right)=\sigma(\rho, j),
$$

where $\sigma(\rho, j)$ is as before the EP rate corresponding to the pair $(\rho, j)$. Then, the analysis of the previous sections can be repeated exactly by replacing there $L$ with $\tilde{L}(\rho, j)=L\left(\rho, j+h_{\rho}\right)$. The new Lagrangian $\tilde{L}$ inherits all the properties of $L$, and gives rise to dynamical activity $\tilde{\psi}(\rho, j), \tilde{L}(\rho, 0)$ as before. We thus obtain bounds on the entropy production rate $\sigma^{*}$ from the same reasonings as above. The simplest quadratic scenario is

$$
L(\rho, j)=\frac{1}{4}\left[j-h_{\rho}-A F(\rho)\right] \frac{1}{\rho A}\left[j-h_{\rho}-A F(\rho)\right]
$$

for force $F$ and matrix $A$. Then indeed, $\tilde{L}(\rho,-j)-\tilde{L}(\rho, j)=$ $j F(\rho)$ is the physical entropy production rate. For example, for the Kramers equation with energy $E(q, p)=p^{2} / 2+$ $U(q)$ and with symplectic matrix $K$, we have $h_{\rho}=$ $-\rho K\left[\nabla E+(-f(q), 0)^{T}\right]$ and $F(\rho)=-\rho\{\nabla(E+\log \rho)+$ $\left.[-f(q), 0]^{T}\right\}$, with $\nabla=\left(\partial_{q}, \partial_{p}\right)$. Then, the symmetry (17) 
holds for the dynamics $\dot{q}=p, \dot{p}=-\partial_{q} U(q)+f(q)-$ $\gamma p+\sqrt{2 \gamma} \xi_{t}$, with $\xi_{t}$ standard white noise and for $A$ having matrix element $A_{p p}=\gamma$ and zeroes elsewhere. Detailed calculations leading to Eq. (17) are found in Ref. [28].

Conclusion.-The theory of dynamical macroscopic fluctuations provides a unifying framework for refinements of the second law, and extends previous work on thermodynamic uncertainties to underdamped and possibly nonMarkov processes. Our arguments point to what really matters in all that, which is the dynamical activity allowing the production of entropy. Interestingly, the system must allow for fluctuating currents contributing both to lower and to higher EP, thus showing negative contributions to the variable $\mathrm{EP}$ rate, in order for the expected $\mathrm{EP}$ rate to be strictly positive.

We were able to add the nontrivial case of underdamped diffusions to the analysis. Not treated here are time-periodic systems and quantum systems. For the first, similar ideas still apply with a time-dependent Lagrangian in Eq. (4) but there appears an extra contribution to the dynamical activity in terms of the dispersion of the time variation, not worked out here but illustrated in, e.g., Ref. [29]. For quantum systems the theory of dynamical fluctuations is not sufficiently developed and non-Markovian effects may prove essential. It is not clear, for example, how intrinsic timesymmetric quantum fluctuations would contribute to the dynamical activity even at zero temperature.

[1] S. R. de Groot and P. Mazur, Non-Equilibrium Thermodynamics (North- Holland, Wiley, Amsterdam, New York, 1962).

[2] J. Schnakenberg, Network theory of behavior of master equation systems, Rev. Mod. Phys. 48, 571 (1976).

[3] C. Maes and K. Netočný, Static and dynamical nonequilibrium fluctuations, C.R. Phys. 8, 591 (2007).

[4] L. Bertini, A. De Sole, D. Gabrielli, G. Jona-Lasinio, and C. Landim, Macroscopic fluctuation theory, Rev. Mod. Phys. 87, 593 (2015).

[5] M. D. Donsker and S. R. Varadhan, Asymptotic evaluation of certain Markov process expectations for large time I, Commun. Pure Appl. Math. 28, 1 (1975).

[6] C. Maes, K. Netočný, and B. Wynants, On and beyond entropy production; the case of Markov jump processes, Markov Processes Relat. Fields 14, 445 (2008).

[7] C. Maes, K. Netočný, and B. Wynants, Steady state statistics of driven diffusions, Physica A (Amsterdam) 387A, 2675 (2008).

[8] L. Bertini, A. De Sole, D. Gabrielli, G. Jona-Lasinio, and C. Landim, Macroscopic fluctuation theory for stationary nonequilibrium states, J. Stat. Phys. 107, 635 (2002).

[9] C. Maes and K. Netočný, The canonical structure of dynamical fluctuations in mesoscopic nonequilibrium steady states, Europhys. Lett. 82, 30003 (2008).
[10] C. Maes, K. Netočný, and B. Wynants, Dynamical fluctuations for semi-Markov processes, J. Phys. A 42, 365002 (2009).

[11] M. I. Freidlin and A. D Wentzell, Random Perturbations of Dynamical Systems, Grundlehren der Mathematischen Wissenschaften (Springer, New York, 1998), Vol. 260.

[12] Jin Feng and T. G. Kurtz, Large Deviations for Stochastic Processes, Mathematical Surveys and Monographs (American Mathematical Society, Providence, RI, 2006).

[13] H. Tasaki, Two theorems that relate discrete stochastic processes to microscopic mechanics, arXiv:0706.1032v1.

[14] C. Maes and K. Netočný, Time-reversal and entropy, J. Stat. Phys. 110, 269 (2003).

[15] C. Maes, From dynamical systems to statistical mechanics: the case of the fluctuation theorem, J. Phys. A 50, 381001 (2017).

[16] A. Mielke, M. A. Peletier, and D. R. M. Renger, On the relation between gradient flows and the large-deviation principle, with applications to Markov chains and diffusion, arXiv:1312.7591.

[17] A. Mielke, D. R. M. Renger, and M. A. Peletier, A generalization of Onsager's reciprocity relations to gradient flows with nonlinear mobility, J. Non-Equil. Thermodyn. 41, 141 (2016).

[18] C. Maes, F. Redig, and M. Verschuere, No current without heat, J. Stat. Phys. 106, 569 (2002).

[19] A. C. Barato and U. Seifert, Thermodynamic Uncertainty Relation for Biomolecular Processes, Phys. Rev. Lett. 114, 158101 (2015).

[20] A. C. Barato and U. Seifert, Universal bound on the Fano factor in enzyme kinetics, J. Phys. Chem. B 119, 6555 (2015).

[21] P. Pietzonka, A. C. Barato, and U. Seifert, Affinity- and topology-dependent bound on current fluctuations, J. Phys. A 49, 34LT01 (2016).

[22] T. R. Gingrich, G. M. Rotskof, and J. M. Horowitz, Inferring dissipation from current fluctuations, J. Phys. A 50, 184004 (2017).

[23] P. Pietzonka, A. C. Barato, and U. Seifert, Universal bounds on current fluctuations, Phys. Rev. E 93, 052145 (2016).

[24] T. R. Gingrich, J. M. Horowitz, N. Perunov, and J. L. England, Dissipation Bounds All Steady-state Current Fluctuations, Phys. Rev. Lett. 116, 120601 (2016).

[25] M. Polettini, A. Lazarescu, and M. Esposito, Tightening the uncertainty principle for stochastic currents, Phys. Rev. E 94, 052104 (2016).

[26] Naoto Shiraishi, Keiji Saito, and Hal Tasaki, Universal trade-off relation between power and efficiency for heat engines, Phys. Rev. Lett. 117, 190601 (2016).

[27] Changbong Hyeon and Wonseok Hwang, A simple proof of the thermodynamic uncertainty relation, Phys. Rev. E 96, 012156 (2017).

[28] R. Kraaij, A. Lazarescu, C. Maes, and M. A. Peletier, Deriving GENERIC from a generalized fluctuation symmetry, arXiv:1706.10115.

[29] T. Demaerel and C. Maes, Activity induced first order transition for the current in a disordered medium, Condens. Matter Phys. 20, 33002 (2017). 